\title{
Influencia de estrategias de lectura en la comprensión lectora de estudiantes V ciclo en 81019 Josefina Gutierrez Fernandez, Chocope 2020
}

\author{
Janet Mabel Garcia Riojas \\ jgarciari@ucvvirtual.edu.pe \\ Dulio Oseda Gago \\ dosedag@unmsm.edu.pe \\ Universidad César Vallejo de Trujillo \\ Trujillo - Perú
}

\section{RESUMEN}

Este artículo presenta la importancia de dos variables fundamentales en la función comunicativa del leer diferentes tipos de textos. El estudio en su objetivo general buscó determinar la correlación entre las estrategias de lectura y la comprensión lectora de estudiantes V ciclo en 81019 Josefina Gutierrez Fernandez, Chocope 2020. La investigación fue de tipo cuantitativa no experimental, transversal, alcance correlacional causal. Se aplicó un cuestionario y una prueba de comprensión lectora a una muestra de 130 estudiantes. A partir del análisis descriptivo, se evidencia un nivel adecuado en las estrategias de lectura y un nivel alto de la comprensión lectora. En resultado inferencial con $r=, 480^{* *}$ (correlación moderada); $r=, 230^{* * *}$ (correlación baja); $r=, 758^{* *}$ (correlación alta); con Sig. Bilateral $<0.01<0,05$. Y presenta una incidencia significativa en dimensiones literal y critica $\mathrm{r} 2=23.4 \%$; $\mathrm{r} 2=8.9 \%$; $\mathrm{r} 2=60.4 \%$; no con la inferencial. Por lo que se concluye que existe una correlación directa positiva fuerte entre las dos variables.

Palabras clave: estrategias de lectura; comprensión lectora; estrategias didácticas; leer. 


\title{
Influence of reading strategies on the reading comprehension of students $\mathrm{V}$ cycle in 81019 Josefina Gutierrez Fernandez, Chocope 2020
}

\begin{abstract}
This article presents the importance of two fundamental variables in the communicative function of reading different types of texts. The study in its general objective sought to determine the correlation between reading strategies and reading comprehension of students V cycle in 81019 Josefina Gutierrez Fernandez, Chocope 2020. The research was quantitative, non-experimental, cross-sectional, causal correlational scope. A questionnaire and a reading comprehension test were applied to a sample of 130 students. From the descriptive analysis, an adequate level of reading strategies and a high level of reading comprehension are evidenced. In inferential result with $\mathrm{r}=.480 * *$ (moderate correlation); $\mathrm{r}=.230 * *$ (low correlation); $\mathrm{r}=.758 * *$ (high correlation); with Sig. Bilateral $<0.01<0.05$. And it presents a significant incidence in literal and critical dimensions $\mathrm{r} 2=23.4 \% ; \mathrm{r} 2=8.9 \% ; \mathrm{r} 2=60.4 \%$; not with the inferential. Therefore, it is concluded that there is a strong positive direct correlation between the two variables.
\end{abstract}

Keywords: reading strategies; reading comprehension; teaching strategies; read.

Artículo recibido: 10. Junio. 2021 Aceptado para publicación: 16. Julio. 2021 Correspondencia: jgarciari@ucvvirtual.edu.pe Conflictos de Interés: Ninguna que declarar 


\section{INTRODUCCIÓN}

A nivel mundial niños y adolescentes no alcanzan los niveles mínimos de competencia (NMC) en lectura (Instituto de Estadística de UNESCO) afectando el progreso del objetivo de desarrollo sostenible 4. En América Latina y el Caribe el 36\% de niños y adolescentes no leen de manera competente.

Según las pruebas PISA realizadas en el año 2018, el Perú alcanzó el 401 de promedio ubicándolo en el puesto 64 en las capacidades de lectura, es decir, una calificación inferior a la del promedio de países de la Organización para la Cooperación y el Desarrollo Económico (OCDE).

De acuerdo a la última Evaluación Censal de Estudiantes (ECE) de Educación Primaria en el año 2019, se tiene que a nivel nacional los resultados indican una medida promedio no muy alentadores, lo que significa que los estudiantes en ambos grados no lograron los aprendizajes esperados en lectura (MINEDU, 2020).

El problema de la comprensión lectora V ciclo en Institución Educativa 81019 Josefina Gutierrez Fernandez, Chocope 2020, se ha identificado y analizado los niveles de logro obtenidos en los resultados de lectura en la ECE 2019 para el 2do y 4to grados; los mismos que la ubicaron "por debajo del promedio del grupo" en relación a otras instituciones similares (Ministerio de Educación, 2020).

Por lo tanto, se formula la siguiente interrogante de investigación: ¿Existe influencia entre estrategias de lectura en la comprensión lectora de estudiantes V ciclo en 81019 Josefina Gutierrez Fernandez, Chocope 2020? Problemas específicos: ¿Existe influencia entre estrategias de lectura en comprensión literal de la comprensión lectora de estudiantes V ciclo en 81019 Josefina Gutierrez Fernandez, Chocope 2020?, ¿Existe influencia entre estrategias de lectura en comprensión inferencial de la comprensión lectora de estudiantes V ciclo en 81019 Josefina Gutierrez Fernandez, Chocope 2020?, ¿Existe influencia entre estrategias de lectura en comprensión criterial de la comprensión lectora de estudiantes V ciclo en 81019 Josefina Gutierrez Fernandez, Chocope 2020?

Esta investigación se justifica en los aspectos siguientes: Práctica, de los resultados que se obtenga se le alcanzará a la institución educativa para que tome decisiones que le ayude a mejorar el uso de estrategias de lectura y la comprensión lectora. Metodológica, se hizo uso de fuentes bibliográficas, herramientas e instrumentos para 
investigar las variables de estudio. Social, por su pertinencia con la institución educativa los resultados van a contribuir a superar los niveles de comprensión lectora de los estudiantes.

El objetivo general: Determinar la influencia entre estrategias de lectura en comprensión lectora de estudiantes V ciclo en 81019 Josefina Gutierrez Fernandez, Chocope 2020. Y como objetivos específicos, determinar la influencia entre estrategias de lectura en comprensión literal de la comprensión lectora de estudiantes V ciclo en 81019 Josefina Gutierrez Fernandez, Chocope 2020; determinar la influencia entre estrategias de lectura en comprensión inferencial de la comprensión lectora de estudiantes $\mathrm{V}$ ciclo en 81019 Josefina Gutierrez Fernandez, Chocope 2020 y determinar la influencia entre estrategias de lectura en comprensión criterial de la comprensión lectora de estudiantes V ciclo en 81019 Josefina Gutierrez Fernandez, Chocope 2020.

La hipótesis general: Existe influencia entre estrategias de lectura en comprensión lectora de estudiantes V ciclo en 81019 Josefina Gutierrez Fernandez, Chocope 2020; de manera específica, se tiene: Existe influencia entre estrategias de lectura en comprensión literal de la comprensión lectora de estudiantes V ciclo en 81019 Josefina Gutierrez Fernandez, Chocope 2020; Existe influencia entre estrategias de lectura en comprensión inferencial de la comprensión lectora de estudiantes V ciclo en 81019 Josefina Gutierrez Fernandez, Chocope 2020; Existe influencia entre estrategias de lectura en comprensión criterial de la comprensión lectora de estudiantes $\mathrm{V}$ ciclo en 81019 Josefina Gutierrez Fernandez, Chocope 2020.

Respecto a los fundamentos teóricos y conceptuales en las variadas fuentes científicas que se han podido revisar, sobre la variable Estrategias de lectura que de acuerdo a Solé (1998) 0sostiene que, son los diversos procedimientos de orden superior que se unen al proceso que realiza el lector al desarrollar sus competencias lectoras. Separa el proceso en la lectura con tres momentos, que son las dimensiones de este estudio: Estrategias antes de la lectura, este momento consiste en que el lector descifra los propósitos por el cual va a leer un determinado texto, contar con un previo conocimiento luego de conocer el título principal, y hacer sus predicciones sobre lo que tratará y formule sus hipótesis. Estrategias durante la lectura, en este momento se realiza el acto de leer el texto, aquí el lector se hace preguntas acerca de la coherencia que contiene, va aclarando sus dudas respecto a alguna idea y su significado para que la pueda 
comprender. Estrategias después de la lectura, en este último momento en que se termina la lectura del texto, el lector puede realizar actividades que le permitan recordar lo leído cómo hacer un resumen, responder a preguntas, un mapa conceptual, entre otros que le ayuden a interpretar. Además, recomienda que al iniciar una lectura se dispongan a contestar preguntas en cada una de las etapas del proceso (Solé, 2005).

Se refuerza en la teoría del Aprendizaje por descubrimiento de Bruner: En ella se induce al estudiante a una participación activa en el proceso de aprendizaje donde pone de manifiesto todo lo que sabe para solucionarlas, así mismo, el aspecto ambiental permitirá desafíos frecuentes que favorecen transferir los aprendizajes (Bruner, 19682011).

La teoría sociohistórico-cultural de Lev Vygotsky, señala que el aprendizaje se desarrolla dentro del contexto en donde se relaciona la persona en su vida social; cómo se lleve con las personas que le rodean y los recursos que la cultura le otorga (Vygotsky, 1993).

Así mismo, (Caparella, 2001) señala que las estrategias didácticas son importantes en la manera que el docente las haga funcionar con la finalidad de que el estudiante se de cuenta que la lectura es fundamental en su desempeño académico y personal. Nogales (2000), las estrategias didácticas son un aserie de métodos, prácticas, que son usadas por los docentes para realizar explicaciones, motivar, hacer comprender y dar orientaciones en la ejecución de la enseñanza y el aprendizaje en el aula.

La variable Comprensión lectora se sustenta en la definición de Cassany (2012) que señala, son un conjunto de pasos que realiza el lector durante su experiencia de lectura que le interesa entender, como producto de leer e interactuar con el texto. Y está dimensionada por tres niveles: comprensión literal (Es la acción que realiza el lector al memorizar lo que contiene en el texto), comprensión inferencial (Es la comprensión de lo que está implícito en el texto, de lo que se supone o hace para anticiparse sobre el tema de la lectura, es decir, expresa sobre qué trata el tema) y comprensión criterial (Es comprender un texto, descubrir el propósito del autor; qué postura asume como lector ante el texto y sus razones), de acuerdo a (Cassany, 2006).

Se basa en la teoría del Aprendizaje significativo de Ausubel quien señala que el aprendizaje se da cuando todo lo aprendido lo relaciona de manera autónoma con los 
conocimientos que ya conoce y lo que aprende, es decir, construye significados de acuerdo a como comprende la realidad Ausubel (1976 - 2002).

Todo lo que cada uno de los estudiantes interioriza, dialoga en su espacio escolar resulta a partir de los diversos textos escritos (Valladores, 1998). Todas las interacciones que se dan entre el lector y el texto son en un contexto en el que se encuentran los dos (Jones, 1998). Los mensajes que se transfieran en su lengua de origen, facilitarán la expresión y la comprensión de textos (Mayor, 2001).

\section{ESTRATEGIAS METODOLÓGICAS O MATERIALES Y MÉTODOS}

El estudio realizado es del tipo de investigación aplicada, con un enfoque cuantitativo que, al utilizarlo con la escala de Likert, permitió ejecutar el análisis estadístico de los datos que se obtuvieron. Fue con un diseño no experimental del tipo transversal, correlacional causal que, según Hernández, Fernandez y Baptista (2010), es correlacional causal porque se asocian variables con la finalidad de conocer las relaciones entre dos o más variables, categorías o conceptos en un determinado momento. En términos correlacionales o a veces en relación causa-efecto.

\section{El diseño que se incluirá en la presente investigación será:}

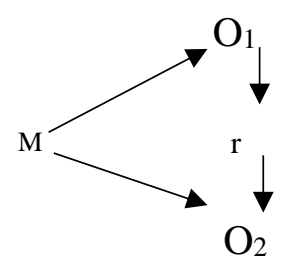

\section{Dónde:}

$\mathbf{M}=$ Muestra: $\quad$ Estudiantes V ciclo 81019 JGF, Chocope 2020

$\mathbf{O}_{1}$, Variable 1: Estrategias de lectura

$\mathbf{O}_{2}$, Variable 2: Comprensión Lectora

$\mathbf{r}=$ relación entre las dos variables.

La población objeto de estudio del presente trabajo estuvo comprendida por los 160 estudiantes V ciclo en 81019 Josefina Gutierrez Fernandez, Chocope 2020. La población está conformada por el universo o la totalidad de elementos a los cuales se les investiga (Toscano, 2018). La muestra de estudio estuvo considerada en un total de 130 estudiantes. El muestreo que se aplicó fue el probabilístico aleatorio simple, por cuanto García et al. (2013), explica que, en este tipo, todos los participantes tienen la misma 
probabilidad de ser elegidos, a diferencia del no probabilístico que es elegido por medio de una fórmula aleatoria.

\section{RESULTADOS Y DISCUSIÓN}

Se muestran los resultados descriptivos de estrategias de lectura y comprensión lectora y resultados inferenciales de ambas y sus dimensiones.

Tabla 1: Categorías de estrategias de lectura

\begin{tabular}{cccccc}
\hline & & f & \% & \% válido & \% acumulado \\
\hline \multirow{2}{*}{ Válido } & $\begin{array}{c}\text { Inadecuado } \\
\text { Regular }\end{array}$ & 5 & 3.8 & 3.8 & 3.8 \\
& Adecuado & 60 & 46.2 & 46.2 & 50.0 \\
& & 65 & 50.0 & 50.0 & 100.0 \\
Total & & 130 & 100.0 & 100.0 & \\
\hline
\end{tabular}

Nota. Data de Estrategias de lectura

\section{Interpretación:}

Se evidencia que en las estrategias de lectura predomina el nivel adecuado con el $50 \%$ (65 estudiantes), sigue el nivel regular con el $46.2 \%$ (60 estudiantes) y con nivel inadecuado el $3.8 \%$ (5 estudiantes). Se interpreta que estas estrategias son adecuadas para los estudiantes al contribuir en la información que leen. Estos resultados se sustentan por Solé (1998) quien señala que las estrategias de lectura son los diversos procedimientos de orden superior que se unen al proceso que realiza el lector al desarrollar sus competencias lectoras.

Tabla 2: Categorías de comprensión lectora

\begin{tabular}{cccccc}
\hline & & $\mathbf{f}$ & $\%$ & \% válido & \% acumulado \\
\hline \multirow{2}{*}{ Válido } & Bajo & 0 & 0 & 0 & 0 \\
& Medio & 2 & 1.5 & 1.5 & 1.6 \\
& Alto & 128 & 98.5 & 98.5 & 100.0 \\
Total & 130 & 100.0 & 100.0 & \\
\hline \multicolumn{5}{c}{ Nota . Data de Comprensión lectora }
\end{tabular}

\section{Interpretación:}

Sobre la comprensión lectora el nivel alto muestra predominio con el $98.5 \%$ (128 estudiantes), le sigue el nivel medio con el $1.5 \%$ ( 2 estudiantes) y $0 \%$ en el nivel bajo. 
Por tanto, se interpreta que la comprensión lectora es muy favorable para los estudiantes a fin de que logren la comprensión de lo que leen. Lo que se corrobora con Cassany (2012) quien afirma que la comprensión lectora son procesos que el lector pone en acción durante su experiencia de lectura que le interesa entender, como producto de leer e interactuar con el texto.

Tabla 3: Prueba de Normalidad de Estrategias de Lectura y Comprensión Lectora

\begin{tabular}{lccc}
\hline & \multicolumn{3}{c}{ Kolmogorov-Smirnov $^{\mathrm{a}}$} \\
\cline { 2 - 4 } & Estadístico & $\mathrm{gl}$ & Sig. \\
\hline Ant &, 134 & 130 &, 000 \\
\hline Dur &, 099 & 130 &, 003 \\
\hline Des &, 096 & 130 &, 005 \\
\hline EstLect &, 062 & 130 &, $200^{*}$ \\
\hline Lit &, 250 & 130 &, 000 \\
\hline Inf &, 355 & 130 &, 000 \\
\hline Cri &, 256 & 130 &, 000 \\
\hline ComL &, 167 & 130 &, 000 \\
\hline
\end{tabular}

Nota. Data Estrategias de Lectura y Comprensión Lectora

\section{Interpretación:}

La mayoría de Sig. de Estrategias de Lectura y Comprensión Lectora y dimensiones son $<0.05$, mostrando así una distribución no paramétrica, por lo que para las relaciones entre estas variables y subvariables se usará la fórmula de Rho Spearman.

Tabla 4: Relaciones de Estrategias de Lectura con Comprensión Lectora y sus dimensiones

\begin{tabular}{llcccc}
\hline & Lit & Inf & Cri & ComL \\
\hline EstLect & Coeficiente de correlación &, $230^{* *}$ &, 091 &, $758^{* * *}$ &, $480^{* *}$ \\
\cline { 2 - 6 } & Sig. (bilateral) &, 008 &, 304 &, 000 &, 000 \\
\hline $\mathrm{N}$ & 130 & 130 & 130 & 130 \\
\hline
\end{tabular}

Nota. Data Estrategias de Lectura y Comprensión Lectora

\section{Interpretación:}

Existe relación altamente significativa entre Estrategias de Lectura con Comprensión Lectora y sus dimensiones Literal, y Criterial: $r=, 480^{* *}$ (Correlación moderada); $\mathrm{r}=, 230^{* *}$ (Correlación Baja); r=,758** (Correlación Alta); con Sig. < 0.01. No existe 
relación significativa entre Estrategias de Lectura y dimensión Inferencial de la Comprensión Lectora.

Tabla 5: Influencia de las Estrategias de Lectura en la Comprensión Lectora y sus

Dimensiones

\begin{tabular}{|c|c|c|c|c|}
\hline Modelo & $\mathbf{R}$ & $\begin{array}{c}\mathbf{R} \\
\text { cuadrado }\end{array}$ & $\begin{array}{l}\mathbf{R} \text { cuadrado } \\
\text { ajustado }\end{array}$ & $\begin{array}{c}\text { Error estándar de la } \\
\text { estimación }\end{array}$ \\
\hline EstLect en Lit &, $298^{a}$ & ,089 &, 082 & 1,091 \\
\hline EstLect en Cri &, $777^{\mathrm{a}}$ & ,604 & ,601 & ,488 \\
\hline $\begin{array}{l}\text { EstLect en } \\
\text { ComL }\end{array}$ &, $484^{\mathrm{a}}$ & ,234 & ,228 & 1,845 \\
\hline
\end{tabular}

Nota. Data Estrategias de Lectura y Comprensión Lectora

\section{Interpretación:}

Las Estrategias de Lectura inciden significativamente en la Comprensión Lectora y en sus dimensiones Literal, y Crítica: $\mathrm{r} 2=23.4 \%$; r2=8.9\%; r2=60.4\%; con Sig. $<0.01$.

Las Estrategias de Lectura no inciden significativamente en la dimensión Inferencial de la Comprensión Lectora.

\section{DISCUSIÓN}

Según los estadísticos descriptivos se evidencia que en las estrategias de lectura predomina el nivel adecuado con el $50 \%$ (65 estudiantes), sigue el nivel regular con el $46.2 \%$ (60 estudiantes) y con nivel inadecuado el $3.8 \%$ (5 estudiantes). Se interpreta que estas estrategias son adecuadas para los estudiantes al contribuir en la información que leen.

Estos resultados se sustentan por Solé (1998) quien señala que las estrategias de lectura son los distintos procedimientos de orden superior que se juntan al proceso que ejecuta el lector cada vez que desarrolla sus competencias en lectura. Se corroboran los resultados obtenidos

Sobre la comprensión lectora el nivel alto muestra predominio con el $98.5 \%$ (128 estudiantes), le sigue el nivel medio con el $1.5 \%$ ( 2 estudiantes) y $0 \%$ en el nivel bajo. Por tanto, se interpreta que la comprensión lectora es muy favorable para los estudiantes a fin de que logren la comprensión de lo que leen.

Lo que se corrobora con Cassany (2012) quien nos afirma que la comprensión lectora son un conjunto de pasos que realiza el lector durante su experiencia de lectura que le 
interesa entender, como el producto de leer e interaccionar con el texto. Sustento que se observa con un predominio de nivel alto en la Comprensión lectora de este estudio.

Es también corroborado por Jesen (2005) al señalar que es un proceso por medio del cual la persona que lee construye un significado al interactuar con el texto. La base de la comprensión es una interacción que se produce con el lector y el texto. Estos pasos de relacionar a la información nueva y a la que ya posee es la comprensión. De ello se demuestra que los estudiantes si tienen una buena comprensión lectora.

De acuerdo a la prueba de normalidad de Estrategias de lectura y Comprensión lectora, la mayoría de Sig. de Estrategias de Lectura y Comprensión Lectora y dimensiones son $<0.05$, mostrando así una distribución no paramétrica, por lo que para las relaciones entre estas variables y subvariables se usará la fórmula de Rho Spearman.

Los resultados de correlación se concluyen que existe influencia directa y significativa entre estrategias de lectura y la comprensión lectora con un valor de $0,480 * *$ (correlación moderada) y con una significancia de $0.000<0.05$. Observándose que, a mejor desarrollo de Estrategias de Lectura, mejor será el desarrollo de Comprensión Lectora en comprensión literal y criterial.

Avendaño (2020) menciona en su artículo: Influence of the Reading Strategies of Isabel Solè in the Reading understanding of the educandes of fifth grade of primary. Universidad Mayor de San Marcos, que las estrategias de lectura propuestas por Solé, si mejoraron de manera significativa los niveles de la comprensión lectora en los estudiantes. Se relacionan estos resultados con obtenidos en este estudio, ya que las dos variables Estrategias de lectura en comprensión lectora tienen incidencia significativa.

Del mismo modo Santillana (2017) en su tesis de maestría: Estrategias de aprendizaje y comprensión lectora en estudiantes de sexto grado de la institución educativa $\mathrm{N}^{\mathrm{o}} 7035$, San Juan de Miraflores, 2015. Universidad César Vallejo, tiene como una conclusión que hay una relación positiva de correlación entre sus dos variables, alcanzando un nivel de significancia de 0.05 . Esta investigación se relaciona con este estudio en una correlación positiva y un nivel de significancia menor a 0.05 .

Estos resultados también, son fortalecidos por Bruner (1968-2011) en su teoría del Aprendizaje por descubrimiento, en ella se induce al estudiante a una participación activa en el proceso de aprendizaje. Esto se entiende que al estudiante se le proporciona situaciones para que ponga de manifiesto todo lo que sabe y pueda solucionarlas, así 
mismo, el aspecto ambiental permitirá que en desafíos permanentes le sea de utilidad lograr transferir los aprendizajes. Estas afirmaciones nos orientan a tener en cuenta que las estrategias en conjunto, llevan a descubrir al estudiante aprendizajes en la información que lee con comprensión, partiendo de lo que conoce, como se entiende los resultados obtenidos en la presente investigación.

De acuerdo a los resultados hallados se evidencia que existe una correlación moderada de un $0,230 * *$ en una significación bilateral de $0.01<0,05$ y un R2 de 0,89 entre las estrategias de lectura y la dimensión comprensión literal de la comprensión lectora, existiendo una incidencia significativa.

Se relaciona este resultado con Caparella (2001) quien señala que las estrategias didácticas son importantes en la manera cómo las ponga en funcionamiento el docente con la finalidad que el estudiante pueda darse cuenta que la lectura es primordial en su desempeño académico y personal, además, el que él lea lo llevará a disfrutar de la lectura de distintos tipos de texto. Del mismo modo, Nogales (2000), nos refiere que las estrategias didácticas son una serie de métodos, prácticas, que son tomadas en cuenta por los docentes para realizar explicaciones, motivar, hacer comprender y dar presiones en la ejecución de enseñar y del aprender en el aula.

Nos da fortalecimiento la teoría sociohistórico-cultural de Lev Vygotsky, que fundamenta que el aprendizaje se desarrolla dentro del entorno donde se interrelaciona la persona en su vida social. Va a depender de acuerdo al tipo de relación que lleve con las personas que le rodean y de las condiciones o recursos que la cultura le otorga para basar su pensamiento. De ello, podrá adquirir sus conocimientos, actitudes, ideas, valores, costumbres, manera de interrelacionarse y forma de vivir (Vygotsky, 1993). La comprensión literal al ser trabajada por el docente los estudiantes se estimulan para identificar características (tiempo, hechos, personajes, etc.) en el texto que leen, tal como se puede apreciar en los resultados que los estudiantes respondieron significativamente ante los recursos presentados.

Se tiene del resultado entre estrategias de lectura y dimensión inferencial de la comprensión lectora que, el 0,091 de coeficiente de correlación, por lo tanto, no existe relación significativa entre Estrategias de Lectura y dimensión Inferencial de la Comprensión Lectora; y no hay una incidencia significativa. 
Nos corrobora Burbano, M. (2018) en su artículo: The teaching-learning process of the mother language: The reading comprehension in the students of the primary basic level. Universidad Cienfuegos de Colombia, quién usó varios métodos dentro de ellos la prueba pedagógica a estudiantes y como una de sus conclusiones identificó que los estudiantes quinto grado tienen dificultades significativas en la comprensión lectora. Se relaciona en parte con los resultados que se encontraron por no haber alcanzado un nivel significativo en comprensión inferencial, debido a dificultades en los estudiantes.

Así también, Sierra (2019) en su tesis de maestría: Niveles de comprensión lectora en estudiantes de sexto grado de primaria de una institución educativa pública del Callao. Universidad San Ignacio De Loyola, se pude apreciar que en una de sus conclusiones indica que los estudiantes tuvieron los niveles bajo y medio que demuestra que tienen dificultades para decodificar, construir significados y estructurar esquemas con la información que leen y responden a la comprensión lectora. Se vincula con el resultado obtenido al no tener incidencia significativa en la dimensión comprensión inferencial de la comprensión lectora.

\section{Cuarta}

Existe influencia directa y significativa alta entre estrategias de lectura y la dimensión criterial de la comprensión lectora con un coeficiente de correlación de $0,758^{* *}$ con un Sig. Bilateral de 0,01 menos a 0,05 y un R2 de 60.4\% con lo que se rechaza la hipótesis nula (H0) y se acepta la hipótesis alterna (H1); lo cual se concluye que existe incidencia significativa.

Cutipa (2015) en su tesis de maestría: Estrategias del antes, durante y después de la lectura para la comprensión de textos en los estudiantes del quinto grado sección "B" del nivel secundario de la I.E. "San Francisco De Asís", Andarapa, provincia de Andahuaylas, región Apurímac. Arequipa. Perú. Universidad Nacional de San Agustín, llegó a la conclusión que los estudiantes comprenden lo que leen en nivel inferencial y criterial al dar juicios de valor. Su resultado es coincidente parcialmente con los resultados obtenidos en el presente estudio puesto que, en el nivel criterial alcanzó una mejora, con alta incidencia significativa, pero, difiere en el nivel inferencial que no evidencia una mejora significativa.

Erràzuriz et àl. (2020) en su artículo Do we comprehend texts more if we like to read?: Reading attitudes and performance of students of public schools in Araucanía, Chile. 
Pontificia Universidad Católica de Chile, expresa como conclusión que hay baja comprensión y desempeño lector en general y que conforme se promueven de grado su nivel de comprensión lectora disminuye significativamente. El estudio mencionado se relaciona en parte con la investigación porque el nivel de la comprensión inferencial no alcanzó un nivel satisfactorio.

Montoya (2018) en su artículo llamado: Secuencias didácticas para la enseñanza de la lectura. Una reflexión. Universidad de los Andes, nos dice que su investigación la realizó en niños del $5^{\circ}$ grado de primaria; arribó a algunas conclusiones como que las estrategias de lectura permiten al estudiante partiendo del texto, leer el contexto, hacer predicciones, y relacionar con lo que ya sabe; así también, menciona que la variedad de textos permitió que los educandos enriquezcan su vocabulario y desarrollen competencias básicas para comprender mejor. La investigación se relaciona con el resultado correlacional y de influencia que se logró en un nivel altamente significativo en la comprensión criteral.

Se basa en Ausbel (1976-2002) con la teoría del Aprendizaje Significativo, quien señala que el aprendizaje se da cuando todo lo aprendido lo relaciona de manera autónoma con los conocimientos que ya conoce y lo que aprende, es decir, construye significados de acuerdo a como comprende la realidad. Esta relación se desarrolla partiendo de dos aspectos: la acción de construcción aprendiendo y haciendo la interactuación con otros para observarlas, dar atención a sus explicaciones. Esta teoría nos refiere que el estudiante ha comprendido, relacionado lo que ya tenía aprendido con lo que fue construyendo de acuerdo a su comprensión de la realidad presentada (estrategias de lectura, recursos textuales, entre otros), lo cual le favorece obtener niveles altamente significativos, como se evidencia en estos resultados obtenidos en la comprensión criterial.

\section{CONCLUSIÓN O CONSIDERACIONES FINALES}

\section{Primera}

Según los resultados encontrados se concluye que existe influencia directa y significativa entre estrategias de lectura y la comprensión lectora en estudiantes V ciclo en 81019 Josefina Gutierrez Fernandez, Chocope 2020, con un coeficiente de correlación $0,480 * *$ y un coeficiente R2 de 0.234 , observándose que, a mejor desarrollo de Estrategias de Lectura, mejor será el desarrollo de Comprensión Lectora. 


\section{Segunda}

De acuerdo a los resultados hallados se evidencia que existe una correlación moderada de un $0,230^{* *}$ en una significación bilateral de $0.01<0,05$ y un $\mathrm{R} 2$ de 0,89 entre las estrategias de lectura y la dimensión literal de la comprensión lectora, existiendo una incidencia significativa.

\section{Tercera}

Se tiene del resultado entre estrategias de lectura y dimensión inferencial de la comprensión lectora que, el 0,091 de coeficiente de correlación, por lo tanto, no existe relación significativa entre Estrategias de Lectura y dimensión Inferencial de la Comprensión Lectora; y no hay una incidencia significativa.

\section{Cuarta}

Existe influencia directa y significativa alta entre estrategias de lectura y la dimensión criterial de la comprensión lectora con un coeficiente de correlación de $0,758^{* *}$ con un Sig. Bilateral de 0,01 menos a 0,05 y un R2 de $60.4 \%$ con lo que se rechaza la hipótesis nula $(\mathrm{H} 0)$ y se acepta la hipótesis alterna (H1); lo cual se concluye que existe incidencia significativa.

\section{LISTA DE REFERENCIAS}

Ausubel, D. P. (1976). Psicología evolutiva. Un punto de vista cognoscitivo. México.

Avendaño, Y. M. (2020). Influence of the Reading Strategies of Isabel Solè in the Reading understanding of the educandes of fifth grade of primary. Revista Scientific Electronic Library Online. https://doi.org/10.1590/SciELOPreprints.884

Bamberger, R. (1975). La promoción de la lectura. París. España.

Bruner, J. S. (1968). El proceso de la educación. México.

Bruner, J. S. (2011). Aprendizaje por descubrimiento. México.

Burbano, M. E. (2018). The teaching-learning process of the mother language:

The reading comprehension in the students of the primary basic level.

Colombia. Revista Conrado.

http://scielo.sld.cu/scielo.php?script=sci_arttext\&pid=S19908644201800050031 $\underline{0 \& \text { lang=es }}$

Caparella, C. (2001). La organización escolar. España. 
Cassany, D. (2012). En línea: Leer y escribir en la red. Anagrama. Barcelona.

Cassany, D. (2006). Tras las líneas sobre la lectura contemporánea. Anagrama. Barcelona.

Colomer, T. (2002). Lecturas sobre Lecturas. Bogotá. Colombia.

Cutipa, L. M. (2015). Estrategias del antes, durante y después de la lectura para la comprensiòn de textos en los estudiantes del quinto grado sección " $B$ ” del nivel secundario de la I.E. "San Francisco De Asis", Andarapa, provincia de Andahuaylas, región Apurimac. Arequipa. Perù.

Errázuriz , M.C., Fuentes, L., Cocio, A., Davison, O., Becerra, R. y Aguilar, P. (2020). Do we comprehend texts more if we like to read?: Reading attitudes and performance of students of public schools in Araucania, Chile. Revista Brasileira de Lingüística Aplicada.https://doi.org/10.1590/1984-6398202015350

Flavell, J. (1993). El desarrollo cognitivo. Madrid: Visor Distribuciones.

Garcìa-Garcìa, M. A., Arèvalo, M.A. y Hernàndez, C.A. (2018). La comprensión lectora y el rendimiento escolar. Cuadernos de Linguìstica Hispànica, (32), 155-174. https://www.redalyc.org/articulo.oa?id=322258748008

Hernandez, R. y Fernandez, C. (2006). Metodología de la investigación. Madrid. España.

Herrada-Valverde, G. y Herrada, R. I. (2017). Análisis del proceso de Comprensión lectora de los estudiantes desde el modelo construcción- integración. Perfiles Educativos. https://doi.org/10.22201/iisue.24486167e.2017.157.58448

Jesen, E. (2005). Cerebro y Aprendizaje. Madrid.

Jones, B. (1998). Estrategias para enseñar y aprender. México.

León, J. (1991). La mejora de la comprensión lectora. Madrid.

Mayor, J. (2001). Estrategias Metacognitivas. Aprender a aprender y Aprender a pensar. México.

Ministerio de Educación. (2020). ¿Cómo rinden nuestros estudiantes en la escuela? Lima. Perú.

Murillo, W. (2008). La investigación científica.

Monereo, Carles, Castelló, Monserrat, Clariana Mercè, Palma Monserrat y Pérez, María L. (1999). Estrategias de Enseñanza y Aprendizaje. Barcelona. España. 
Montoya, M. E. (2018). Secuencias didácticas para la enseñanza de la lectura.

Una reflexión. Revista Educere, (74), 69-76. https://www.redalyc.org/articulo.oa?id=35657597007

Sánchez, H. y Reyes, C. (1996). Metodología y diseños en la investigación científica. Lima: editorial Mantaro.

Santillana, G. (2017). Estrategias de aprendizaje y comprensión lectora en estudiantes de sexto grado de la institución educativa $N^{o} 7035$, San Juan de Miraflores. 2015. Lima. Perú.

Sierra, C. (2019). Niveles de comprensión lectora en estudiantes de sexto grado de primaria de una institución educativa pública del Callao. Lima. Perú.

Schraw, G. y Bruning, R. (1996). “Readers `implicit models of Reading”, Reading Research Quarterly, (31), 290-305.

Solé, I. (2005). El proceso de la comprensión lectora. Barcelona. España.

Solé, I. (1998). Estrategias de Lectura. Barcelona. España.

Toscano. Frey (2018). Metodología de la investigación. Guía práctica con las preguntas más frecuentes. Colombia.

Valladares Rodríguez, Otto. (1998). Comunicación integral. Bases técnicas y desarrollo de competencias comunicativas. México.

Varas, A. (2015). 7 pasos para elaborar una tesis. Editorial Macro.

Vygotsky, L. (1993). Connotaciones y aplicaciones de la psicología sociohistórica en la educación. Editorial Aiqué.

Weinstein, E. y Mayer, R. E. (1986). The teaching of learning strategies. Nueva York. 